\title{
Differences in sialic acid density in pathogenic and non-pathogenic Aspergillus species
}

\author{
Julie A. Wasylnka, ${ }^{1}$ Megan I. Simmer ${ }^{2}+$ and Margo M. Moore ${ }^{2}$ \\ Author for correspondence: Margo M. Moore. Tel: +1 604291 3441. Fax: +1 6042913496. \\ e-mail:mmoore@sfu.ca
}

Department of Molecular Biology and Biochemistry ${ }^{1}$ and Department of Biological Sciences 2 , Simon Fraser University, Burnaby, B.C., Canada V5A 156
Aspergillus fumigatus is a ubiquitous soil fungus that causes invasive lung disease in the immunocompromised host. The structure of the conidial wall has not been well characterized although it is thought that adhesins present on the surface are involved in attachment of the conidia to host lung cells and proteins, which is a prerequisite for the establishment of infection. Negatively charged carbohydrates on the conidial surface have been previously identified as the molecules responsible for attachment of conidia to extracellular matrix proteins. The aim of this research was to identify carbohydrates on the conidial surface that contribute to its negative charge. Direct chemical analysis and indirect binding assays have demonstrated that $A$. fumigatus possesses sialic acids on the conidial surface. Pre-treatment of $A$. fumigatus conidia with sialidase decreased binding of a sialic acid-specific lectin, Limax flavus agglutinin (LFA), to the conidial surface and decreased adhesion of conidia to the positively charged polymer poly L-lysine. Two other sialic acid-specific lectins, Maackia amurensis agglutinin and Sambucus nigra agglutinin, exhibited negligible binding to $A$. fumigatus conidia indicating that $2,3-\alpha$ - and 2,6- $\alpha$-linked sialic acids are not the major structures found on the conidial surface. Mild acid hydrolysis and purification of conidial wall carbohydrates yielded a product that had the same $R_{\mathrm{F}}$ as the Neu5Ac standard when analysed by high-performance thin-layer chromatography. A density of $6.7 \times 10^{5}$ sialic acid residues per conidium was estimated using a colorimetric assay. Conidia grown on a minimal medium lacking sialic acid also reacted with LFA, indicating that sialic acid biosynthesis occurs de novo. Sialic acid biosynthesis was shown to be regulated by nutrient composition: the density of sialic acids on the surface of conidia grown in minimal media was lower than that observed when conidia were grown on rich, complex media. It has previously been shown that pathogenic Aspergillus species adhere to basal lamina proteins to a greater extent than non-pathogenic Aspergillus species. To determine whether the expression of sialic acid on the conidial surface was correlated with adhesion to basal lamina, conidia from other non-pathogenic Aspergillus species were tested for their reactivity towards LFA. Flow cytometric analysis demonstrated that $A$. fumigatus had a significantly greater sialic acid density than three non-pathogenic Aspergillus species. Sialic acids on the conidial wall may be involved in adhesion to fibronectin, a component of the basal lamina, as binding of $A$. fumigatus conidia to fibronectin was strongly inhibited in the presence of a sialylated glycoprotein.

Keywords: Aspergillus fumigatus, invasive aspergillosis, lectin, flow cytometry, adhesion

† Present address: Department of Microbiology and Immunology, University of British Columbia, Vancouver, B.C., Canada.

Abbreviations: HPTLC, high-performance thin-layer chromatography; LFA, Limax flavus agglutinin; MAA, Maackia amurensis agglutinin; MALDI, matrixassisted laser desorption ionization; Neu5Ac, $\mathrm{N}$-acetylneuraminic acid; Neu5Gc, $\mathrm{N}$-glycolylneuraminic acid; SNA, Sambucus nigra agglutinin. 


\section{INTRODUCTION}

Aspergillus species are saprophytic fungi found in soil, water and decaying organic matter. Of the 200 species in the genus only a few can cause disease including Aspergillus fumigatus, Aspergillus flavus, Aspergillus niger, Aspergillus nidulans and Aspergillus terreus; however, over $90 \%$ of all cases are caused by $A$. fumigatus (Denning, 1998). In the normal host, Aspergillus species can cause a wide spectrum of diseases ranging from allergic manifestations and superficial infections (Bodey \& Vartivarian, 1989), to a lifethreatening invasive disease known as invasive aspergillosis. Initiation of infection begins with the inhalation of conidia, the infectious particles, which adhere to and germinate in the lung tissue (Latge, 1999). Hyphal invasion of the lung epithelium is followed by dissemination to other organs (Broderick et al., 1996). Risk factors for invasive aspergillosis are prolonged neutropenia and/or long-term administration of high dose corticosteroids (Denning, 1998).

The frequency of invasive aspergillosis has increased 14fold in the past decade (Groll et al., 1996), mainly due to the greater numbers of bone marrow and organ transplant recipients (Wald et al., 1997), more aggressive cytotoxic chemotherapy used to treat cancer patients (Bodey et al., 1992) and the emergence of the human immunodeficiency syndrome (HIV-AIDS) (Minamoto et al., 1992). There are currently only two antifungal agents used to treat the disease, amphotericin B and itraconazole, and their success rate is only $34 \%$, even when used prophylactically (Denning, 1998). The increase in fungal infections worldwide and the lack of satisfactory antifungal therapy makes it critical to understand the mechanisms by which Aspergillus species adhere to and colonize the lung tissue.

Previous work in our laboratory has demonstrated that A. fumigatus conidia bind significantly better than those of other Aspergillus species to intact lung cell basal lamina and fibronectin (Wasylnka \& Moore, 2000). Investigation of the mechanism of conidial binding determined that negatively charged carbohydrates occurring on the conidial surface may play a role in the adhesion of the conidia to host basal lamina (Wasylnka \& Moore, 2000).

Sialic acids are members of a family of 9-carbon carboxylated monosaccharides that are the major ionogenic compounds in many cell types (Schauer, 1982). All compounds are thought to be derived from $\mathrm{N}$-acetylneuraminic acid (Neu5Ac) with the most common modifications being the oxidation of the $\mathrm{N}$-acetyl group to form $\mathrm{N}$-glycolylneuraminic acid (Neu5Gc) and acetylation of the hydroxyl groups at the $4,7,8$ or 9 position (Varki, 1992). In most glycoconjugates, the sialic acids are terminal or subterminal non-reducing sugars and are attached by $2,3-\alpha$ or $2,6-\alpha$ linkages to underlying galactose residues.

Sialic acids have been shown to be important in several aspects of microbial pathogenesis. Many viruses bind to their host via sialic acids on the epithelial cell surface.
For example, influenza $\mathrm{A}$ and $\mathrm{B}$ viruses recognize sialic acid whereas influenza $\mathrm{C}$ viruses bind exclusively to 9O-acetylated sialic acids (Varki, 1997). Incorporation of sialic acids into the capsules of some pathogenic bacteria aids in evading host defences by inhibiting the direct activation of the alternative complement pathway (Wessels et al., 1992). More recently, sialic acids have been identified in the cell walls of several pathogenic fungi including Candida albicans (Soares et al., 2000), Cryptococcus neoformans (Rodrigues et al., 1997), Paracoccidioides brasiliensis (Soares et al., 1998) and, to a lesser degree, Pneumocystis carinii (De Stefano et al., 1990). In pathogenic fungi, the biological function of these carbohydrates has not yet been determined but several studies have provided evidence that, as in other microbial pathogens, sialic acids may play a role in host recognition and in the evasion of host defences. For example, one report found that removal of sialic acids from Cry. neoformans yeast cells enhanced their susceptibility to phagocytosis by mouse macrophages (Rodrigues et al., 1997). The authors postulated that these sialic acids might give the fungus a protective advantage in the host during the early stages of infection (Rodrigues et al., 1997).

Preliminary analysis of the carbohydrate structures on the conidia of A. fumigatus has shown the presence of $\mathrm{D}-$ galacto-D-mannans (Barreto-Bergter et al., 1981). Sialic acid substitution was not detected by these investigators because of the conditions used in the isolation procedures. Therefore, the aims of this study were: 1 , to determine whether A. fumigatus conidia possess sialoglycoconjugates; 2 , to identify the nature of the glycan linkages; and 3, to determine whether the A. fumigatus cell wall contains greater amounts of sialic acid than the cell walls of three non-pathogenic Aspergillus species.

\section{METHODS}

Aspergillus strains and growth conditions. The following Aspergillus species were used in this study: A. fumigatus ATCC 13073, ATCC 42202 and CHUV (gift from M. Monod, Laboratoire de Mycologie, Centre Hospitalier Universitaire Vaudois, Lausanne, Switzerland); Aspergillus ornatus ATCC 16921 and ATCC 66492; Aspergillus wentii ATCC 10584 and ATCC 1023; and A. auricomus ATCC 16890. All fungi were grown on $\mathrm{YM}$ agar $(0.3 \%, \mathrm{w} / \mathrm{v}$, yeast extract and malt extract; $0.5 \%, \mathrm{w} / \mathrm{v}$, peptone; and $1 \%, \mathrm{w} / \mathrm{v}$, glucose) for $5-14 \mathrm{~d}$ at $28^{\circ} \mathrm{C}$ (except A. ornatus ATCC 66492, which was grown at $24^{\circ} \mathrm{C}$ ) until conidia were fully mature. For some experiments, A. fumigatus ATCC 13073 was grown on a sialic acid-free minimal medium $\left[1 \%, \mathrm{w} / \mathrm{v}\right.$, glucose; $0.52 \mathrm{~g} \mathrm{KCl} \mathrm{l}^{-1}$; $0.52 \mathrm{~g} \mathrm{MgSO}_{4} .7 \mathrm{H}_{2} \mathrm{O} \mathrm{l}^{-1} ; 1.52 \mathrm{~g} \mathrm{KH}_{2} \mathrm{PO}_{4} \mathrm{l}^{-1}$; Hunters trace elements; $0.85 \mathrm{~g} \mathrm{NaNO}_{3} \mathrm{l}^{-1}$; vitamins ( $1 \mathrm{mg}$ nicotinic acid $\mathrm{l}^{-1}$, $2.5 \mathrm{mg}$ riboflavin $\mathrm{l}^{-1}, 2 \mathrm{mg}$ pantothenic acid $\mathrm{l}^{-1}, 0.5 \mathrm{mg}$ pyridoxine $\mathrm{l}^{-1}, 0.01 \mathrm{mg}$ biotin $\mathrm{l}^{-1}, 0.2 \mathrm{mg}$-aminobenzoic acid $1^{-1}$ ) and $1.5 \%, \mathrm{w} / \mathrm{v}$, agar]. Conidia were harvested by flooding the plate with PBS, pH 7.4/0.05\% (v/v) Tween 20 and gently scraping with a sterile cotton swab. The suspension was then vortexed for $30 \mathrm{~s}$ to break up the chains of conidia and hyphal fragments were removed by filtration through sterile glass wool. Conidia were pelleted by centrifugation $(1500 \mathrm{~g}, 2 \mathrm{~min})$, resuspended in PBS and counted using a haemocytometer. 
Sialidase treatment. Conidia were harvested from a mature plate of $A$. fumigatus ATCC 13073 and $2 \times 10^{7}$ conidia were resuspended in $50 \mathrm{mM}$ sodium acetate buffer, $\mathrm{pH} 5.0$ containing 0 or $1 \cdot 1 \mathrm{U}$ Clostridium perfringens $2,3-\alpha-, 2,6-\alpha-, 2,8-\alpha-$ sialidase (Calbiochem) and incubated for $6 \mathrm{~h}$ at $37^{\circ} \mathrm{C}$.

Biotinylation of lectins. LFA (Calbiochem), S. nigra agglutinin (SNA; Sigma) or M. amurensis agglutinin (MAA; Sigma) (all $1 \mathrm{mg}$ ) were incubated with a 20 molar excess of n-hydroxysuccinimidobiotin long chain (Pierce) in $1 \mathrm{ml} 0 \cdot 1 \mathrm{M}$ sodium phosphate buffer, $\mathrm{pH} 7 \cdot 4$ containing $0 \cdot 15 \mathrm{M} \mathrm{NaCl}$, for $60 \mathrm{~min}$ at room temperature. The biotinylated lectins were then dialysed against PBS in a $4 \mathrm{ml}$ Ultrafree membrane filter with an $M_{\mathrm{r}} 10000$ cut-off membrane (Millipore).

\section{Lectin labelling.}

Sialidase-treated samples. Untreated or sialidase-treated A. fumigatus conidia were reacted with biotinylated LFA essentially as described by Rodrigues et al. (1997) with the following modifications. Cells were blocked for $60 \mathrm{~min}$ with PBS $/ 10 \%$ goat serum and incubated with biotinylated LFA at $50 \mu \mathrm{g} \mathrm{ml}^{-1}$ in PBS $/ 10 \%$ goat serum for $60 \mathrm{~min}$; bound lectin was detected by incubation for $60 \mathrm{~min}$ with StreptavidinOregon Green (Molecular Probes) diluted 1:150 in PBS/10\% goat serum. Flow cytometry was performed on a Coulter EPICS Elite Esp flow cytometer using a $488 \mathrm{~nm}$ laser for excitation energy and a $550 \mathrm{~nm}$ dichroic filter capturing an emission band at $525 \mathrm{~nm}$ : 10000 events were recorded for each sample. Samples were also viewed with an Olympus VANOX AHBS3 microscope equipped with epifluorescence at $1000 \times$ magnification. Bright field and fluorescent images for each sample were captured with a Sony 950 camera using Eclipse (Empix Imaging) image capturing software.

Determination of sialic acid linkage. To determine the sialic acid linkages in A. fumigatus conidia, an aliquot of $2 \times 10^{7}$ cells was lectin labelled as described above using $50 \mu \mathrm{g}$ biotinylated LFA, SNA or MAA ml ${ }^{-1}$ and then analysed by flow cytometry or fluorescence microscopy as described above.

Competition of LFA binding by sialic acid. An aliquot of $3.6 \times 10^{7}$ A. fumigatus ATCC 13073 conidia was added to poly L-lysine coated coverslips for $60 \mathrm{~min}$ and reacted with biotinylated LFA in PBS $/ 5$ \% goat serum containing 0 or $100 \mathrm{mM} \mathrm{Neu} 5 \mathrm{Ac}$ (Rose Scientific). The remainder of the protocol was as described above. Samples were mounted with ProLong mounting media (Molecular Probes) and analysed by fluorescence microscopy.

Lectin labelling of different Aspergillus species. Conidia from $A$. fumigatus ATCC 13073, ATCC 42202 and CHUV; A. ornatus ATCC 16921 and ATCC 66492; A. wentii ATCC 10584 and ATCC 1023; and A. auricomus ATCC 16890 were collected, and $1 \times 10^{7}$ conidia were labelled with biotinylated LFA and processed for flow cytometry analysis as described above.

Isolation of sialic acids from $\boldsymbol{A}$. fumigatus conidia. Sialic acids from A. fumigatus conidia were isolated as described by Reuter \& Schauer (1994) with minor modifications. Briefly, dry cells $(5 \cdot 5 \mathrm{~g})$ were resuspended in $20-30 \mathrm{ml} 88 \%$ formic acid for $60 \mathrm{~min}$ at $70^{\circ} \mathrm{C}$. The suspension, which should contain any $\mathrm{O}$-acetylated sialic acids, was cooled on ice and centrifuged for $10 \mathrm{~min}$ at $1500 \mathrm{~g}$. The supernatant was removed, and the pellet resuspended in $20-30 \mathrm{ml} 3 \mathrm{M} \mathrm{HCl}$ and incubated at $80^{\circ} \mathrm{C}$ for $60 \mathrm{~min}$ to remove all remaining sialic acids. This suspension was cooled and centrifuged as described above. The supernatants from both the formic and hydrochloric acid digests were then ultracentrifuged for $30 \mathrm{~min}$ at $50000 \mathrm{~g}$ and $4{ }^{\circ} \mathrm{C}$, and the ultrasupernatants were lyophilized overnight. The dried residues were resuspended in $4 \mathrm{ml}$ cold, deionized, distilled $\mathrm{H}_{2} \mathrm{O}\left(\mathrm{ddH}_{2} \mathrm{O}\right)$ and then dialysed against
$\mathrm{ddH}_{2} \mathrm{O}$ in a $5000 \mathrm{Da}$ ( $\mathrm{HCl}$ digest) or $10000 \mathrm{Da}(\mathrm{HCOOH}$ digest) molecular mass cut-off Ultrafree-4 centrifugal filter (Millipore). The diffusate was passed over a Dowex 50WX8 (100-200 mesh) cation exchange column (hydrogen ion form; Sigma) and washed with $3-4$ bed vols cold $\mathrm{ddH}_{2} \mathrm{O}$. The eluate and washings were collected, lyophilized, resuspended in $5 \mathrm{ml}$ cold $\mathrm{ddH}_{2} \mathrm{O}$ and the $\mathrm{pH}$ adjusted to 4.0 with $\mathrm{NH}_{4} \mathrm{OH}$. This solution was then passed over a Dowex 2X8 (200-400 mesh) anion exchange column (formate form; Sigma), washed with $10 \mathrm{ml}$ cold dd $\mathrm{H}_{2} \mathrm{O}$ and then eluted with $20 \mathrm{ml} 1 \mathrm{M}$ formic acid. Fractions of $1.5 \mathrm{ml}$ were collected and lyophilized. The dried residues were resuspended in $40 \mu \mathrm{lddH_{2 }} \mathrm{O}$ and $10 \mu \mathrm{l}$ was spotted onto HPTLC (high-performance thin-layer chromatography) plates (Mandel Scientific) along with $5 \mu \mathrm{l} \mathrm{Neu5Ac}$ and Neu5Gc (Sigma) standards (at $1 \mathrm{mg} \mathrm{m}^{-1}$ ) and run in 1propanol/1 $\mathrm{M} \mathrm{NH} \mathrm{NH}_{3} / \mathrm{H}_{2} \mathrm{O}$ (6:2:1 by vol.). Plates were developed by spraying with orcinol/ $\mathrm{HCl}$ spray reagent $(81.4 \mathrm{ml}$ $37 \% \mathrm{HCl}, 0 \cdot 2 \mathrm{~g}$ orcinol (Sigma), $2 \mathrm{ml} 1 \% \mathrm{FeCl}_{3}$ ) diluted $3: 1$ with $\mathrm{ddH}_{2} \mathrm{O}$ and then heated at $180{ }^{\circ} \mathrm{C}$ for $20 \mathrm{~min}$.

Colorimetric quantitation of sialic acids. For colorimetric quantitation, $4 \times 10^{10}$ conidia were hydrolysed with $0 \cdot 1 \mathrm{M}$ trichloroacetic acid for $3 \mathrm{~h}$ at $80^{\circ} \mathrm{C}$. The suspension was cooled and centrifuged at $9000 \mathrm{~g}$ for $10 \mathrm{~min}$. The supernatant was then treated with $0 \cdot 1 \mathrm{M} \mathrm{HCl}$ for $1 \mathrm{~h}$ at $80^{\circ} \mathrm{C}$ and dialysed overnight

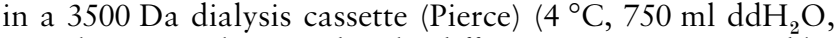
two changes). The next day the diffusate was concentrated by rotary vacuum until the final volume was $250 \mathrm{ml}$ and this was lyophilized to dryness. Ion-exchange chromatography was performed as described above. To further purify the sialic acids from contaminants, anion exchange fractions were streaked onto HPTLC plates and the sialic acid containing bands scraped off and extracted with $\mathrm{ddH}_{2} \mathrm{O}$ followed by $1: 1$ $\mathrm{ddH}_{2} \mathrm{O} /$ methanol. These extracts were lyophilized by speed vacuum, resuspended in $\mathrm{ddH}_{2} \mathrm{O}$ and analysed by colorimetric quantitation as described by Reuter \& Schauer (1994) by measuring the absorbance at $572 \mathrm{~nm}$ of samples reacted with the orcinol $/ \mathrm{Fe}^{3+} / \mathrm{HCl}$ reagent.

Matrix-assisted laser desorption ionization (MALDI) MS. Mass spectra of purified sialic acids were obtained using a MALDI mass spectrometer from PerSeptive Biosystems with an accelerating voltage of $20 \mathrm{keV}$. An aliquot of purified sialic acids $(2.5 \mu \mathrm{l})$ was mixed with $0.5 \mu \mathrm{l}$ matrix $(10 \mathrm{mg} 2,5$ dihydroxybenzoic acid $\mathrm{ml}^{-1}$ ) and laser ionization focused around areas of good crystallization.

Adhesion to poly L-lysine. Sialidase-treated or untreated $A$. fumigatus conidia were added to poly L-lysine (Sigma) coated coverslips in 24 well plates at a concentration of $1 \times 10^{7}$ conidia $\mathrm{ml}^{-1}$ for $60 \mathrm{~min}$ at room temperature. Conidia were fixed with $\mathrm{PBS} / 4 \%(\mathrm{w} / \mathrm{v})$ paraformaldehyde, $\mathrm{pH} 7.4$ for $60 \mathrm{~min}$ and then coverslips were mounted onto slides. Samples were viewed with an Olympus Vanox AHBS3 microscope at a $1000 \times$ magnification. Sample enumeration was performed by collecting images from five random fields (Sony 950 video camera) and the number of conidia per field was calculated by the computer program Eclipse.

Adhesion assays. Experiments were performed as described previously (Wasylnka \& Moore, 2000). Mucin (Type I-S, Sigma), asialomucin or sialic acid were co-incubated with peroxidase-labelled conidia at the indicated concentrations.

Miscellaneous chemicals and supplies. Vibrio cholerae sialidase was obtained from Calbiochem and Arthrobacter ureafaciens sialidase was from Sigma. All chemicals were reagent grade or better unless specifically noted. Asialomucin was a generous gift from Dr J. Esko (Division of Cellular and Molecular Medicine, University of California, San Diego, CA, USA). 
Statistics. Differences in sialic acid densities between Aspergillus species were analysed by a one-way Kruskal-Wallis test for non-parametric data using JMP (SAS Institute). The Student's $t$ test was used for statistical analysis of other data. Values shown are mean data from three independent experiments (unless otherwise stated). A $P$ value of $<0.05$ was considered significant.

\section{RESULTS AND DISCUSSION}

\section{A. fumigatus conidia have sialic acids on their surface}

We have previously shown that negatively charged carbohydrates present on the conidial surface mediate adhesion of A. fumigatus to basal lamina proteins. This finding, along with the recent identification of sialic
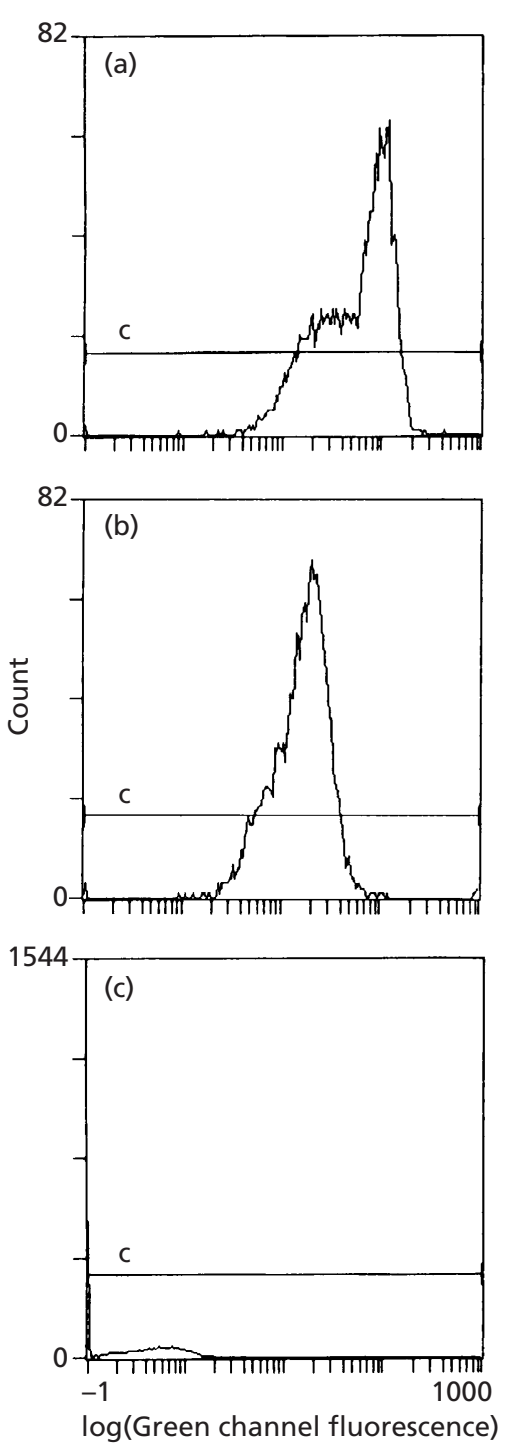

Fig. 1. LFA labelling of sialidase-treated or untreated $A$. fumigatus conidia. A. fumigatus conidia were untreated $(a, c)$ or sialidase treated (b) and then incubated with biotinylated LFA and Streptavidin-Oregon Green $(a, b)$ or StreptavidinOregon Green alone (c) and analysed by flow cytometry.
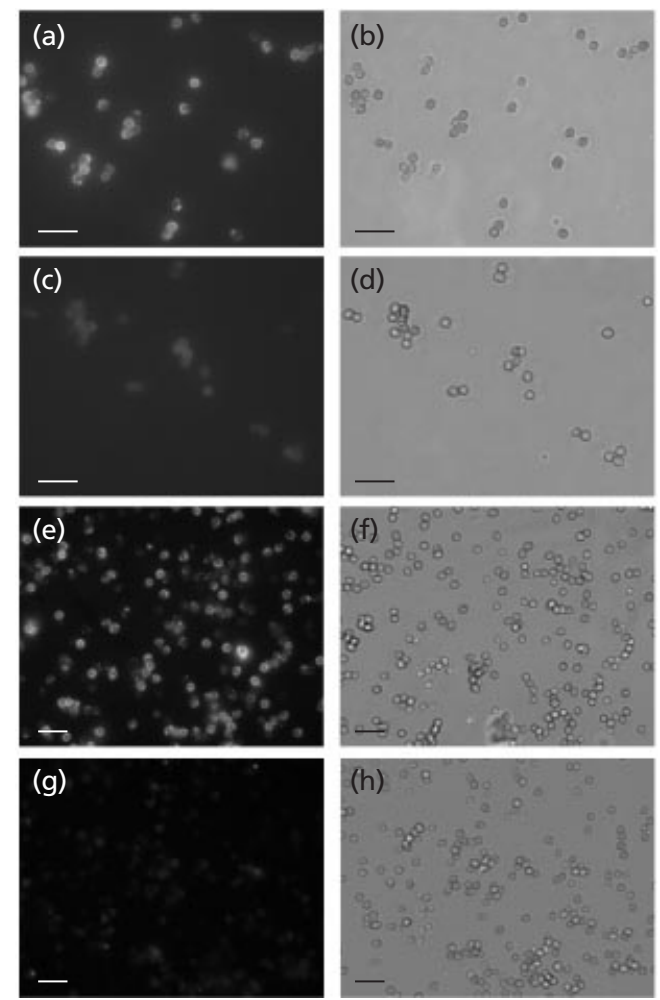

Fig. 2. Epifluorescence microscopy of LFA-labelled conidia demonstrates existence of sialic acids. Untreated (a) or sialidasetreated (c) conidia were incubated with biotinylated LFA and Streptavidin-Oregon Green. Untreated conidia were also incubated with biotinylated LFA in the absence (e) or presence (g) of $100 \mathrm{mM}$ Neu5Ac followed by Streptavidin-Oregon Green. The corresponding differential interference contrast images are shown $(b, d, f, h)$. Bars, $10 \mu \mathrm{m}$. This figure is representative of two independent experiments.

acids in other pathogenic fungi prompted the investigation as to whether A. fumigatus conidia also possessed sialic acids. In earlier reports of A. fumigatus cell wall structure (Barreto-Bergter et al., 1981; Hearn \& Sietsma, 1994), sialic acids were not detected, which may have been a result of the carbohydrate purification scheme used. In addition, the study by Hearn \& Sietsma (1994) investigated hyphal wall preparations, which may contain slightly different carbohydrate structures than conidia. For this reason we used sialic acid-specific lectins to detect sialic acids, since these proteins are very sensitive and specific for particular sialic acid derivatives (Varki, 1997).

A. fumigatus conidia were incubated with biotinylated LFA lectin, which reacts with any sialic acid linkage, (Schauer et al., 1995) and then with a secondary fluorophore, Streptavidin-Oregon Green. A. fumigatus conidia were strongly fluorescent (compared to the sample which received Streptavidin-Oregon Green only), indicating the presence of sialic acids on the conidial surface (Fig. 1a). Analysis by flow cytometry (Fig. 1) and epifluorescence (Fig. 2a-d) of sialidasetreated and untreated conidia showed that pre-treatment 


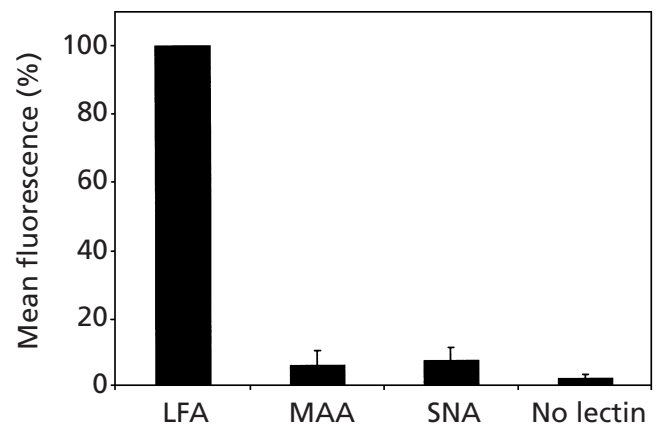

Fig. 3. Reactivity of $A$. fumigatus conidia with different sialic acid-specific lectins. $A$. fumigatus conidia were incubated with biotinylated LFA, SNA, MAA or no lectin, followed by Streptavidin-Oregon Green and analysed by flow cytometry. The histogram of flow cytometry data expresses the mean fluorescence (from three independent experiments) of MAA, SNA and no lectin as a percentage of the LFA fluorescence.

of conidia with Clo. perfringens sialidase significantly reduced the fluorescent signal. Three independent experiments using flow cytometry showed that sialidasetreated conidia had $48 \pm 18 \%$ of the mean fluorescence when compared to the controls (set at $100 \%$ ). We found that removal of sialic acids was only achieved at high concentrations of the Clo. perfringens sialidase $(1 \cdot 1 \mathrm{U})$. Lower concentrations of Clo. perfringens sialidase, $V$. cholerae sialidase or $A$. ureafaciens sialidase were ineffective at cleaving the sialic acids (data not shown).

Additional proof that the reaction of LFA was specific for sialic acid was obtained from lectin-competition experiments. Conidia were incubated with biotinylated LFA in the presence and absence of $100 \mathrm{mM}$ sialic acid and then with Streptavidin-Oregon Green. The fluorescence of the conidia was almost eliminated when sialic acid was added with LFA, compared to the fluorescence observed with LFA alone (Fig. 2e-h). Therefore, the results from the sialidase treatment and the lectin-competition experiment both indicate that the LFA was specifically binding to sialic acids on the $A$. fumigatus conidial cell wall.

To determine the glycosidic linkage of the sialic acids, we reacted $A$. fumigatus conidia with lectins that bind specifically to different sialic acid linkages. Conidia were incubated with biotinylated LFA, MAA or SNA. MAA reacts with Neu5Ac2,3- $\alpha$-Gal1,4- $\beta$-GlcNAc and SNA reacts with Neu5Ac2,6- $\alpha-\mathrm{Gal} / \mathrm{GalNAc}$ (Schauer et al., 1995). The extent of MAA and SNA binding to $A$. fumigatus conidia was much lower than that observed with LFA: LFA-treated conidia were 13 times more fluorescent than SNA-treated conidia and 16 times more fluorescent than MAA-treated conidia as determined by flow cytometry (Fig. 3). To confirm that both SNA and MAA were functional and adequately biotinylated, the lectins were used as probes for binding to sialylated glycoproteins fetuin (MAA) and fibronectin (SNA) immobilized onto nitrocellulose. Both SNA and MAA reacted equally with these glycoproteins (data not

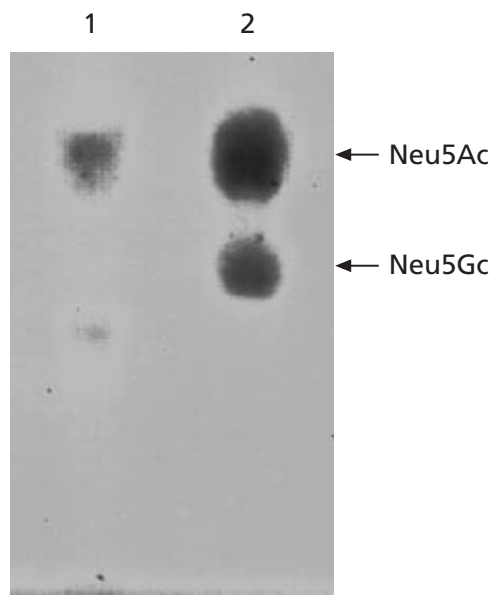

Fig. 4. HPTLC of sialic acids from purified $A$. fumigatus conidia hydrolysate. An HPTLC plate was spotted with purified hydrolysate of $A$. fumigatus conidia (1) or sialic acid standards $(5 \mu \mathrm{g})(2)$ and developed with the orcinol spray reagent.

shown), confirming that the lack of reactivity was due to the structure of the conidial carbohydrates. Since 2,6- $\alpha$ sialic acid has been the predominant sialic acid structure identified in other pathogenic fungi (Rodrigues et al., 1997; Soares et al., 1998, 2000), the lack of reactivity with SNA was surprising. However, filamentous fungi such as Aspergillus may have novel sialic acids that are not found in yeast-like fungi such as Candida and Cryptococcus. In summary, the lack of reactivity of $A$. fumigatus conidia with SNA and MAA suggests that 2,3- $\alpha$ - or 2,6- $\alpha$-linked sialic acids represent a minority of the sialic acid structures present on the A. fumigatus conidial cell walls. Alternatively, the sialic acid may be substituted in such a way that reduces SNA or MAA binding or that other sialic acid linkages, in particular $2,8-\alpha$ or 2,9- $\alpha$, may be present in A. fumigatus conidia. However, since there are no lectins that bind to these structures, this cannot be proven without a more detailed chemical analysis of purified sialic acids.

\section{Identification of sialic acids in A. fumigatus by chemical purification}

To provide direct chemical evidence for the presence of sialic acids on the surface of A. fumigatus, conidia were hydrolysed under mildly acidic conditions and then purified by ion-exchange chromatography using standard protocols (Reuter \& Schauer, 1994; Rodrigues et al., 1997). A spot that had the same $R_{\mathrm{F}}$ value as the Neu5Ac standard was identified by development with the orcinol reagent following HPTLC of the purified hydrolysate (Fig. 4). MALDI MS analysis identified a major peak at $m / z 310$, which is the $(\mathrm{M}+\mathrm{H})^{+}$peak characteristic of Neu5Ac (Fig. 5). These data provide direct chemical evidence for the existence of sialic acids in A. fumigatus and support the results of the lectinbinding experiments. A colorimetric quantitation assay of the purified fraction determined that the sialic acid 


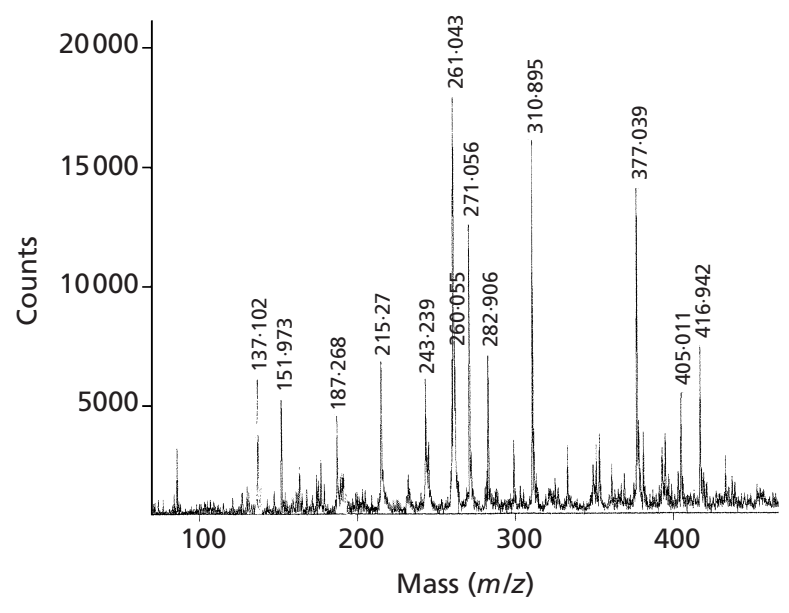

Fig. 5. MALDI MS analysis of purified sialic acid from $A$. fumigatus conidia. An aliquot of purified $A$. fumigatus sialic acid $(2.5 \mu \mathrm{l})$ was mixed with $0.5 \mu \mathrm{l}$ 2,5-dinitrobenzoic acid matrix and run on a PerSeptive Biosystems MALDI mass spectrometer. The $310(\mathrm{M}+\mathrm{H})^{+}$peak is a major component of the sample. $\mathrm{m} / \mathrm{z}$ fragments lower than 260 are due to the matrix. Higher molecular mass fragments are trace amounts of other unknown sugars.

content of our sample was $13.8 \mu \mathrm{g}$ in $4 \times 10^{10}$ conidia, or $6.7 \times 10^{5}$ residues per cell. Using bovine fetuin as a control, our yield of sialic acid with this assay after hydrolysis and chromatography was approximately $50 \%$. Therefore, the actual number of sialic acid residues per conidium may be greater than observed. This value is similar to sialic acid densities found in other fungal species: Fonsecaea pedrosoi, $1.2 \times 10^{5}$ cell $^{-1}$; Par. brasiliensis, $3.7 \times 10^{6}$ cell $^{-1}$; Cry. neoformans, $3 \cdot 0 \times 10^{6}$ cell $^{-1} ;$ Can . albicans, $2 \cdot 8 \times 10^{6}$ cell $^{-1}$ and Sporothrix schenkii, $2 \times 10^{6}$ cell $^{-1}$ (Alviano et al., 1999).

\section{Expression of sialic acids under different growth conditions}

The protozoan parasite Trypanosoma cruzi can transfer sialic acids from sialic acid donors in the host onto mucin-like proteins on its surface using a parasite transsialidase enzyme (Burleigh \& Andrews, 1995). In contrast, all fungi that have been studied synthesize sialic acids de novo (Alviano et al., 1999). Therefore, we investigated whether A. fumigatus could synthesize sialic acids when grown on a chemically defined medium without sialic acid. Conidia grown on minimal medium or YM medium were incubated with biotinylated LFA and then with Streptavidin-Oregon Green. Observation of the samples by epifluorescence microscopy demonstrated that conidia grown on sialic acid-free minimal medium reacted with LFA (data not shown). This result confirms that $A$. fumigatus can synthesize sialic acids de novo. However, quantitation of sialic acids by flow cytometric analysis showed that the density of sialic acids from conidia grown on minimal medium was significantly less than that of conidia grown on YM

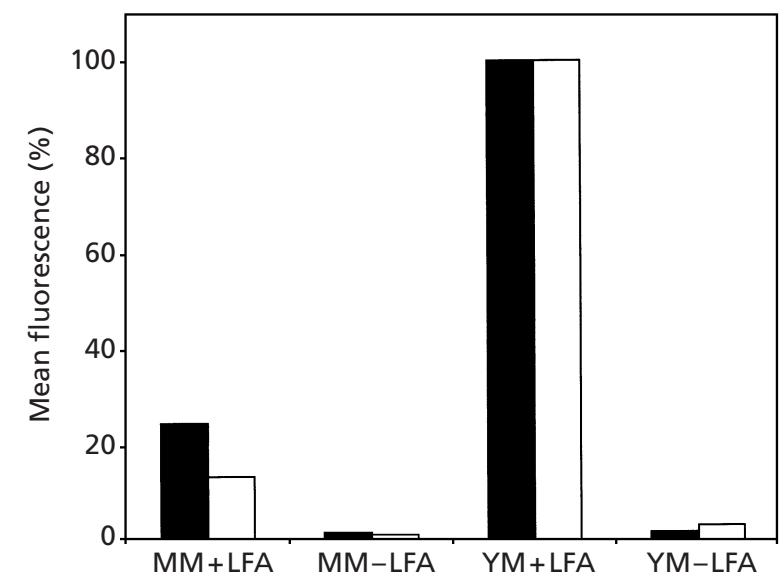

Fig. 6. Expression of sialic acid from conidia grown in minimal media (MM) or complex media (YM). Flow cytometric analysis of conidia grown on MM or YM after incubation with biotinylated LFA and Streptavidin-Oregon Green (+LFA) or with Streptavidin-Oregon Green alone (-LFA). The histogram of the flow cytometry data expresses the mean fluorescence of MM-grown conidia as a percentage of the YM-grown conidia fluorescence. The values from two independent experiments are shown (white bars, expt 1; black bars, expt 2).

medium (Fig. 6). Therefore, in environments of limiting nutrient availability A. fumigatus synthesizes less Neu5Ac, and/or less Neu5Ac is expressed on the conidia surface. Reactivity of conidia grown on minimal medium with SNA and MAA was negligible (data not shown). Therefore, the expression of sialic acids is regulated by the components in the growth medium. The observed difference in sialic acid expression may occur because complex media contain cofactors that stimulate de novo synthesis to a greater extent than minimal medium does. For example, sialyltransferase enzymes catalyse the transfer of CMP-Neu5Ac; thus the formation of the sugar nucleotide may have been limiting due to a low concentration of CTP in our minimal media (Gilbert et al., 1998). Since our ultimate goal is to define the role of sialic acids in the virulence of A. fumigatus, it is more appropriate to grow $A$. fumigatus on a complex medium (such as YM) that is more representative of their natural environment. Thus, it is anticipated that the density of sialic acids expressed during growth in complex media is more comparable to the levels present in naturally occurring infectious conidia. Further experiments are required to determine whether there are any differences between the structures of sialic acids in conidia grown on complex medium versus minimal medium.

\section{Adhesion to poly L-lysine}

Sialic acids are the major anionogenic compounds in the cell surfaces of many species (Schauer, 1982). To determine whether sialic acids play such a role in $A$. fumigatus, conidia were treated with Clo. perfringens sialidase or buffer alone, added to poly L-lysine coated coverslips and bound spores were enumerated with an 
automated counting program. In the first experiment $(n$ $=3$ ), untreated conidia adhered to poly L-lysine at a density of $121 \pm 8$ conidia per field. Pre-treatment of conidia with $\bar{C}$ lo. perfringens sialidase reduced the adhesion of conidia to poly L-lysine by $29 \% \quad(87 \pm 18$ conidia per field) $(P<0.05)$. In a separate experiment, we observed a smaller decrease $(15 \%)$ in adhesion following sialidase treatment of conidia; the value of the buffer-treated sample was $83 \pm 5$ conidia per field whereas the sialidase-treated sample was $71 \pm 6$ conidia per field $(P<0 \cdot 05)$. These data suggest that the carboxylic acid groups of sialic acid are responsible for a significant portion of the electronegative charge on the conidia. The cell surface of a eukaryotic cell contains many other anionic groups including phosphate and sulfate groups, as well as carboxyl groups of acidic proteins and other polysaccharides, so that even with complete removal of sialic acids from the cell surface, other negatively charged groups remain that can adhere to the poly L-lysine polymer. Treatment of A. fumigatus conidia with Clo. perfringens sialidase removed approximately $50 \%$ of the sialic acids (Fig. 1), therefore the remaining sialic acid residues may be sufficient to allow binding to poly L-lysine. Other researchers have reported somewhat larger effects of sialidase on the adhesion of other fungi to poly L-lysine. For example, treatment of Cry. neoformans resulted in a $35 \%$ decrease in adhesion to poly L-lysine (Rodrigues et al., 1997) and a $55 \%$ decrease was observed in Can. albicans (Soares et al., 2000).

\section{Conidia of pathogenic Aspergillus species possess greater amounts of sialic acid than non-pathogenic species}

Previous work in our laboratory has shown that $A$. fumigatus conidia bind to purified fibronectin and intact basal lamina significantly better than other non-pathogenic Aspergillus species (Wasylnka \& Moore, 2000). The biological function of sialic acids in pathogenic fungi is still unknown; however, they are important virulence factors in other micro-organisms such as T. cruzi (Burleigh \& Andrews, 1995) and Neisseria meningitidis (Vogel et al., 1996). To determine whether the density of sialic acids may be correlated with adhesion to basal lamina in Aspergillus, we measured the density of sialic acid on conidia from four species of Aspergillus. In addition to three strains of A. fumigatus, three non-pathogens were also studied: A. wentii (two strains), A. ornatus (two strains) and A. auricomus (one strain). A. fumigatus is the most common cause of invasive aspergillosis (Denning, 1998), while A. wentii, $A$. ornatus and A. auricomus have never been documented in human disease (Pitt, 1994). Adhesion to basal lamina has been previously measured in three of these four species (Wasylnka \& Moore, 2000).

Conidia from all four Aspergillus species were collected from agar plates and incubated with biotinylated LFA followed by Streptavidin-Oregon Green. Analysis by fluorescence microscopy provided preliminary evidence that all three A. fumigatus strains contained greater
Table 1. Sialic acid density on conidia of pathogenic and non-pathogenic Aspergillus species

Conidia from all Aspergillus strains tested were collected and resuspended in PBS. An aliquot of $1 \times 10^{7}$ conidia were fixed with $\mathrm{PBS} / 4 \%$ paraformaldehyde and incubated sequentially with $\mathrm{PBS} / 150 \mathrm{mM} \mathrm{NH}_{4} \mathrm{Cl}, \mathrm{PBS} / 10 \%$ goat serum, $100 \mu \mathrm{g}$ biotinylated LFA ml-1 and then with a 1:100 dilution of Streptavidin-Oregon Green. Samples were adjusted to $5 \times 10^{6}$ conidia $\mathrm{ml}^{-1}$ and analysed on a Coulter EPICS Elite Esp flow cytometer using a $488 \mathrm{~nm}$ laser for excitation energy and a $550 \mathrm{~nm}$ dichroic filter capturing an emission band at $525 \mathrm{~nm}$.

\begin{tabular}{|lc|}
\hline Strain & Fluorescence $^{*}$ \\
\hline A. fumigatus CHUV & $226 \cdot 4$ \\
A. fumigatus ATCC 42202 & $213 \cdot 8$ \\
A. fumigatus ATCC 13073 & $168 \cdot 4$ \\
A. auricomus ATCC 16890 & $48 \cdot 4 \dagger$ \\
A. wentii ATCC 1023 & $24 \cdot 0 \dagger$ \\
A. wentii ATCC 10584 & $12 \cdot 7 \dagger$ \\
A. ornatus ATCC 16921 & $25 \cdot 2 \dagger$ \\
A. ornatus ATCC 66492 & $25 \cdot 6 \dagger$ \\
\hline
\end{tabular}

"Sample/control of the mean log fluorescence channel (controls received Streptavidin-Oregon Green but no LFA).

† Statistically different from A. fumigatus strains as determined by the Kruskal-Wallis test. The data are representative of two independent experiments.

amounts of sialic acids than the other Aspergillus species tested (data not shown). Therefore, the experiment was repeated and the conidia analysed by flow cytometry to quantitatively measure sialic acid density in all four species. Because differences in the shape and size of conidia can affect the data spread in flow cytometry, each species was labelled with both LFA and Streptavidin-Oregon Green (sample fluorescence) and also with Streptavidin-Oregon Green alone as a control. By dividing the mean of the control fluorescence by the value of the sample fluorescence, a mean sample value was obtained which enabled a direct comparison between species. The mean fluorescence of A. fumigatus ATCC 42202 and CHUV was four times greater than the $A$. auricomus strain, 10 times greater than both $A$. ornatus strains and the A. wentii ATCC 1023 strain and 20 times greater than A. wentii ATCC 10584 (Table 1). The mean fluorescence of the other A. fumigatus strain, ATCC 13073, was three times greater than the $A$. auricomus strain, six times greater than both $A$. ornatus strains and the $A$.wentii ATCC 1023 strain and 13 times greater than A. wentii ATCC 10584 (Table 1). Statistical analysis of the mean fluorescence using the KruskalWallis test demonstrated that the mean fluorescence of all the A. fumigatus strains was significantly greater than any of the non- $A$. fumigatus strains $(P<0 \cdot 05)$. Therefore, these experiments showed that the expression of sialic acids on A. fumigatus conidial walls was 3-20 times greater than the conidia of the three nonpathogenic Aspergillus species. In addition, the flow cytometry data comparing sialic acid expression be- 


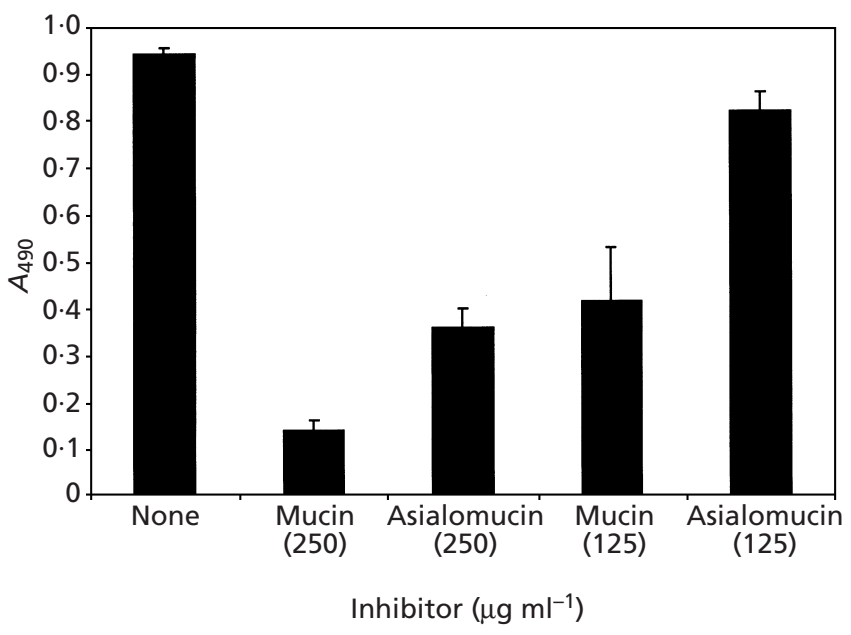

Fig. 7. Binding of $A$. fumigatus conidia to fibronectin in the presence of sialylated carbohydrates. Microtitre plates were coated with fibronectin at $50 \mu \mathrm{g} \mathrm{ml}^{-1}$ for $1 \mathrm{~h}$ at $37^{\circ} \mathrm{C}$ and the next day a standard binding assay was performed. Peroxidaselabelled $A$. fumigatus conidia were added to the plates in the presence of mucin, asialomucin or Neu5Ac at the indicated concentrations for $1 \mathrm{~h}$ at $37^{\circ} \mathrm{C}$. Unbound conidia were washed off with PBS/Tween and the absorbance measured at $490 \mathrm{~nm}$ on a microtitre plate reader (Bio-Tek Instruments) after the addition of the peroxidase substrate. The values are the means \pm standard deviations of triplicate wells and the figure is representative of two independent experiments.

tween Aspergillus species parallels our data on the adhesion of Aspergillus species to basal lamina: all A. fumigatus strains bound to basal lamina with 10-20 fold greater affinity than $A$. ornatus and $A$. wentii strains $(A$. auricomus was not tested) (Wasylnka \& Moore, 2000). Taken together, these data indicate a correlation between sialic acid density and adhesion to basal lamina proteins.

This is the first report of differences in sialic acid expression between pathogenic and non-pathogenic species within a genus. The results indicate that nonpathogenic Aspergillus species synthesize sialic acids; however, either less sialic acid is transported to the cell wall or different glycosylation pathways occur in these species. Since sialic acids are known virulence factors in other micro-organisms, the fact that all A. fumigatus strains tested expressed higher levels of sialic acids on their surfaces than the non-pathogenic strains suggests that sialic acid density may contribute to the virulence of this fungus.

\section{Role of conidial sialic acid in the binding of A. fumigatus to fibronectin and basal lamina}

To confirm that the observed differences in sialic acid density between species correlated with adhesion to basal lamina, we tested the adhesion of A. fumigatus conidia to components of the basal lamina in the presence of sialylated carbohydrates. We used this method because, as stated earlier, sialidase treatment of conidia removes only $50 \%$ of the sialic acids from the spore surface and requires large amounts of enzyme. We first investigated the adhesion of A. fumigatus conidia to purified fibronectin. Conidia were co-incubated with the heavily sialylated glycoprotein mucin $(12 \%$ sialic acid by weight), asialomucin or sialic acid and allowed to adhere to fibronectin-coated microtitre plates. Binding of conidia to immobilized fibronectin was inhibited almost completely (by $85 \%$ ) by $250 \mu \mathrm{g}$ mucin $\mathrm{ml}^{-1}$ and only partially $(62 \%)$ by asialomucin (Fig. 7). Furthermore, at $125 \mu \mathrm{g} \mathrm{ml}^{-1}$, mucin still inhibited binding by $56 \%$, whereas binding to fibronectin in the presence of asialomucin was essentially restored. Therefore, mucin had a dose-dependent inhibitory effect on conidia binding to fibronectin. The inability of asialomucin to strongly reduce binding suggests that it is the sialic acid residues on mucin which are directly involved in the inhibitory effect. Further evidence for the involvement of sialic acids in binding to fibronectin was demonstrated using sialic acid as an inhibitor in binding assays. Coincubation of conidia with $20 \mathrm{mM}$ sialic acid inhibited binding by $90 \%$ (data not shown), although part of the reduction in binding may have been due to the acid $\mathrm{pH}$ of the sialic acid solution ( $\mathrm{pH} 3.5$ at $20 \mathrm{mM}$ ).

These results are similar to those obtained by Bouchara et al. (1997) who demonstrated inhibition of binding of A. fumigatus conidia to fibronectin by mucin; however, their interpretations of the results led to a different conclusion on the mechanism of conidia binding. They concluded that the sialylated glycoprotein mucin was competing with the oligosaccharides on extracellular matrix proteins for a lectin on the conidial surface (Bouchara et al., 1997). However, previous work in our laboratory showed that conidia bound to the heparinbinding domain of fibronectin and neither desialylation nor complete deglycosylation of fibronectin decreased conidial adhesion. Furthermore, negatively charged carbohydrates and high ionic strength buffers inhibited the adhesion of conidia binding to fibronectin (Wasylnka \& Moore, 2000). In the present study we have provided evidence for the existence of sialic acids on the conidial surface of $A$. fumigatus. In addition, we have shown that a sialylated glycoprotein inhibited the adhesion of conidia to fibronectin. Taken together, our results indicate that $A$. fumigatus conidia bind to fibronectin in part via sialoglycoconjugates on the spore surface and that the mechanism of binding is an ionic interaction between the negatively charged sialic acids on the conidial surface and positively charged regions of the fibronectin protein.

We next investigated whether sialic acids on $A$. fumigatus conidia were involved in binding to immobilized basal lamina. Conidia were again coincubated in the presence of mucin, asialomucin or sialic acid and allowed to adhere to basal-lamina-coated microtitre plates. In contrast to the results obtained with fibronectin, mucin (up to $500 \mu \mathrm{g} \mathrm{ml}^{-1}$ ) had no effect on binding of conidia to basal lamina (data not shown). However, as seen with fibronectin, sialic acid was also able to strongly inhibit binding to basal lamina $(69 \%$ at $20 \mathrm{mM}$ ) (data not shown). Therefore, in contrast to 
fibronectin, the binding to intact basal lamina may be more complex; perhaps requiring the participation of surface sialic acids and/or other negatively charged carbohydrates on the spore surface. Nonetheless, it has been suggested that the basal lamina of diseased lungs have increased amounts of fibronectin and other extracellular matrix proteins (Roman \& McDonald, 1997). Therefore, binding of A. fumigatus conidia to fibronectin in vivo may be important in the development of invasive aspergillosis.

\section{ACKNOWLEDGEMENTS}

This work was supported by a grant from the Natural Sciences and Engineering Research Council of Canada (NSERC) to M.M.M. J.A.W. was supported by a NSERC predoctoral scholarship. M.I.S. was a recipient of a Summer Student Works grant from the government of British Columbia.

The authors would like to thank Dr R. Durand (Department of Medical Biophyics, B.C. Cancer Agency, Vancouver, Canada) for use of the Coulter EPICS Elite flow cytometer and helpful discussions, and Denise McDougal for running the samples. The authors also acknowledge Dr B. M. Pinto (Department of Chemistry, Simon Fraser University, Burnaby, Canada) for protocols on the isolation of sialic acids from whole cells.

\section{REFERENCES}

Alviano, C. S., Travassos, L. R. \& Schauer, R. (1999). Sialic acids in fungi: a minireview. Glycoconj J 16, 545-554.

Barreto-Bergter, E., Gorin, P. A. J. \& Travassos, L. R. (1981). Cell constituents of mycelia and conidia of A. fumigatus. Carbohydr Res 95, 205-218.

Bodey, G. P. \& Vartivarian, S. (1989). Aspergillosis. Eur J Clin Microbiol Infect Dis 8, 413-437.

Bodey, G., Bueltmann, B., Duguid, W. \& 11 other authors (1992). Fungal infections in cancer patients: an international autopsy survey. Eur J Clin Microbiol Infect Dis 11, 99-109.

Bouchara, J. P., Sanchez, M., Chevailler, A., Marot-Leblond, A., Lissitzky, J. C., Tronchin, G. \& Chabasse, D. (1997). Sialic aciddependent recognition of laminin and fibrinogen by Aspergillus fumigatus conidia. Infect Immun 65, 2717-2724.

Broderick, L. S., Conces, D. J., Jr, Tarver, R. D., Bergmann, C. A. \& Bisesi, M. A. (1996). Pulmonary aspergillosis: a spectrum of disease. Crit Rev Diagn Imaging 37, 491-531.

Burleigh, B. A. \& Andrews, N. W. (1995). The mechanisms of Trypanosoma cruzi invasion of mammalian cells. Annu Rev Microbiol 49, 175-200.

Denning, D. W. (1998). Invasive aspergillosis. Clin Infect Dis 26, 781-803.

De Stefano, J. A., Cushion, M. T., Puvanesarajah, V. \& Walzer, P. D. (1990). Analysis of Pneumocystis carinii cyst wall. II. Sugar composition. J Protozool 37, 436-441.

Gilbert, M., Bayer, R., Cunningham, A. M., DeFrees, S., Gao, Y., Watson, D. C., Young, N. M. \& Wakarchuk, W. W. (1998). The synthesis of sialylated oligosaccharides using a CMP-Neu5Ac synthetase/sialyltransferase fusion. Nat Biotechnol 16, 769-772.

Groll, A. H., Shah, P. M., Mentzel, C., Schneider, M., JustNuebling, G. \& Huebner, K. (1996). Trends in the postmortem epidemiology of invasive fungal infections at a university hospital. J Infect 33, 23-32.

Hearn, V. M. \& Sietsma, J. H. (1994). Chemical and immunological analysis of the Aspergillus fumigatus cell wall. Microbiology 140, 789-795.

Latge, J. P. (1999). Aspergillus fumigatus and aspergillosis. Clin Microbiol Rev 12, 310-350.

Minamoto, G. Y., Barlam, T. F. \& Vander Els, N. J. (1992). Invasive aspergillosis in patients with AIDS. Clin Infect Dis 14, 66-74.

Pitt, J. I. (1994). The current role of Aspergillus and Penicillium in human and animal health. J Med Vet Mycol 32, 17-32.

Reuter, G. \& Schauer, R. (1994). Determination of sialic acids. Methods Enzymol 230, 168-199.

Rodrigues, M. L., Rozental, S., Couceiro, J. N., Angluster, J., Alviano, C. S. \& Travassos, L. R. (1997). Identification of Nacetylneuraminic acid and its 9-O-acetylated derivative on the cell surface of Cryptococcus neoformans: influence on fungal phagocytosis. Infect Immun 65, 4937-4942.

Roman, J. \& McDonald, J. A. (1997). Fibronectins and fibronectin receptors in lung development, injury and repair. In The Lung: Scientific Foundations, pp. 737-755. Edited by R. G. Crystal \& J. B. West. Philadelphia, PA: Lippincott-Raven.

Schauer, R. (1982). Chemistry, metabolism and biological function of sialic acids. Adv Carbohydr Chem Biochem 40, 131-234.

Schauer, R., Kelm, S., Reuter, G., Roggentin, P. \& Shaw, L. (1995). Biochemistry and the role of sialic acids. In Biology of the Sialic Acids, pp. 7-67. Edited by A. Rosenberg. New York: Plenum.

Soares, R. M. A., Costa e Silva-Filho, F., Rozental, S., Angluster, J., de Souza, W., Alviano, C. S. \& Travassos, L. R. (1998). Anionogenic groups and surface sialoglycoconjugate structures of yeast forms of the human pathogen Paracoccidioides brasiliensis. Microbiology 144, 309-314.

Soares, R. M., de A. Soares, R. M., Alviano, D. S., Angluster, J., Alviano, C. S. \& Travassos, L. R. (2000). Identification of sialic acids on the cell surface of Candida albicans. Biochim Biophys Acta 1474, 262-268.

Varki, A. (1992). Diversity in the sialic acids. Glycobiology 2, 25-40.

Varki, A. (1997). Sialic acids as ligands in recognition phenomena. FASEB J 11, 248-255.

Vogel, U., Hammerschmidt, S. \& Frosch, M. (1996). Sialic acids of both the capsule and the sialylated lipooligosaccharide of Neisseria meningitidis serogroup B are prerequisites for virulence of meningococci in the infant rat. Med Microbiol Immunol 185, 81-87.

Wald, A., Leisenring, W., van Burik, J. A. \& Bowden, R. A. (1997). Epidemiology of Aspergillus infections in a large cohort of patients undergoing bone marrow transplantation. J Infect Dis $175,1459-1466$.

Wasylnka, J. A. \& Moore, M. M. (2000). Adhesion of Aspergillus species to extracellular matrix proteins: evidence for involvement of negatively charged carbohydrates on the conidial surface. Infect Immun 68, 3377-3384.

Wessels, M. R., Haft, H. T., Heggen, L. M. \& Rubens, C. E. (1992). Identification of a genetic locus essential for capsule sialylation in type III group B streptococci. Infect Immun 60, 392-400.

Received 16 October 2000; revised 4 January 2001; accepted 8 January 2001. 